\title{
Nonstoichiometric Polyelectrolyte Complexes: Smart Nanoreactors for Alloy and Multimetallic Catalyst
}

\author{
Md. Shahinul Islam, Won San Choi, and Ha-Jin Lee
}

\begin{abstract}
This work efforts to demonstrates synthesis and potential employment of bimetallic alloy and multimetallic coexisting mixture of metal nanoparticles (NPs) using spherical nonstoichiometric polyelectrolyte complexes (PECs) in aqueous solution, whereas PECs act both as a template to implant metal precursors and nanoreactor for diverse architecture of metal NPs. Alloy-type metal NP-embedded PECs were synthesized by drop wise mixing of two types of oppositely-charged weak PEs preloaded with metal precursors followed by coreduction process. Furthermore, individual metal NPs (e.g. Au, Ag, or Pd) stabilized by PEs were mixed into a different volume ratio and results in bi- or tri-metallic NP-embedded PECs structure where each NPs exist as separate entities rather than alloy or core-shell structure. Catalytic activities of the synthesized materials were tested for reduction of 4-nitrophenol to useable 4-aminophenol. Moreover, they exhibit promising performances for surface enhanced Raman scattering (SERS) activities.
\end{abstract}

Index Terms-Alloy, nanoreactor, nonstoichiometric polyelectrolyte complexes, synergistic effect.

\section{INTRODUCTION}

In recent years, great progresses have been made in the preparation and synergistic catalysis of heterometallic nanoparticles (NPs) such as Au/Ag NPs (alloy/intermetallics etc.) via different synthetic approaches with improved physical and chemical properties compare to monometallic catalysts [1]. Also self-assembly of oppositely-charged polyelectrolytes (PEs) films, capsules [2], brushes [3], or complexes with nanocomposite organic/inorganic shells are of great interest as facile means for creating functional nanomaterials [4]. Here the polymer matrices have a dual role: to provide a scaffold for immobilizing NPs and preventing aggregation, and to serve as a capping agent for limiting the NPs growth [5]. Usually, the encapsulation or immobilization of NPs requires pre-modification with stabilizing and capping agents when the NPs are used for adsorbing onto/into multilayer PE films and/or capsules [4]. Thus far, materials have been assembled on various templates such as $\mathrm{CaCO}_{3}$ particles [6], melamine formaldehyde (MF) particles [7], silica particles [8], and so on. These approaches have some disadvantages such as formation of stable cores with certain surface properties, low incorporation efficiency and the harsh conditions usually used for core decomposition

Manuscript received May 4, 2013; revised July 10, 2013.

Md. S. Islam and H.-J. Lee are with the Jeonju Centre of Korea Basic Science Institute, 664-14 Dukjindong 1-ga, Dukjin-gu, Jeonju, 561-756, Korea (e-mail: shahinul@jbnu.ac.kr, hajinlee@kbsi.re.kr).

W. S. Choi is with the Department of Chemical and Biological Engineering, Hanbat National University, San 16-1, Dukmyoung dong, Yuseong-gu, Daejeon 305-719, Korea (e-mail: choiws@hanbat.ac.kr). (extreme $\mathrm{pH}$ or usage of organic solvents and oxidizing agents) which hinder the functional encapsulation of the macromolecules and confine usage of employed polymers for the other approaches [9].

We therefore, reported on the synthesis of metal NPembedded spherical, stable nonstoichiometric polyelectrolyte complexes (PECs) at room temperature by simple mixing of cationic poly (allylamine hydrochloride) (PAH) and anionic poly(acrylic acid) (PAA) in nonstoichiometric ratio (PAH:PAA=1:0.40) without using sacrificial templates. Subsequently, they were employed as nanoreactors for the synthesis of bimetallic alloy-typed (Au/Ag) NPs by coreduction. Also, post reduction mixing of individual $\mathrm{Au}$, Ag, PdNPs stabilized by PAH and PAA (i.e. PAH-AuNPs, PAA-AgNPs, PAA-PdNPs) leaded to formation of bi-, or tri-metallic heterostructure where each metal NP exists as separate entity rather than core-shell or alloy structure. The as-obtained materials exhibited promising performance for surface enhanced Raman scattering (SERS) and catalytic activities for 4-nitrophenol reduction.

\section{MATERIALS AND METHOD}

Poly (allylamine hydrochloride) ( $\mathrm{PAH}, \mathrm{Mw}=15,000)$, poly (acrylic acid) (PAA, $\mathrm{Mw}=1,800)$, silver nitrate $\left(\mathrm{AgNO}_{3}\right.$, 99.99\%), chloroauric acid $\left(\mathrm{HAuCl}_{4}, 99.99 \%\right)$, palladium nitrate $\left(\mathrm{PdN}_{2} \mathrm{O}_{6}\right)$, 4-nitrophenol (4-NPh), 4-aminothiophenol (ATP), sodium borohydrate $\left(\mathrm{NaBH}_{4}\right)$, sodium hydroxide $(\mathrm{NaOH})$ and nitric acid $\left(\mathrm{HNO}_{3}\right)$ were purchased from Sigma-Aldrich. All chemicals were used without further purification. For all the experiments, the concentration of $\mathrm{Au}$, $\mathrm{Ag}$ and Pd salt was $2.5 \mathrm{mM}$.

Approach I; synthesis of bi-metallic Au/Ag Alloy NP-embedded PECs by coreduction method: For preparing bimetallic alloy PEC (denoted as aPEC- $\mathrm{Au}_{50} / \mathrm{Ag}_{50}$ ) i) $0.5 \mathrm{~mL}$ volume of $\mathrm{HAuCl}_{4}$ solution were added dropwise into a 10 $\mathrm{mL}$ solution of PAH and stirred for 30 min (denoted as PAH-Au). ii) Separately, using $0.5 \mathrm{~mL}$ of $\mathrm{AgNO}_{3}$ solution into $10 \mathrm{~mL}$ of PAA prepared PAA-Ag. Then, the PAA-Ag solution added dropwise into the PAH-Au solution and continued to stir for another 1 hour. Finally, the mixture was thermally reduced at $150^{\circ} \mathrm{C}$ for 1 hour. Likewise, aPECs- $\mathrm{Au} / \mathrm{Ag}=2: 1$ and $\mathrm{aPEC}-\mathrm{Au} / \mathrm{Ag}=1: 2$ etc. were also synthesized. For the synthesis of monometallic AuNPs or AgNPs containing PECs, either PAH-Au or PAA-Ag was mixed with counter ionic PEs, PAA or PAH, respectively.

Approach II; heterostructural multi-metallic NP-embedded PECs by post reduction method: It was separately prepared various metal precursors loaded-PEs i) PAH-Au ii) PAA-Ag and iii) PAA-Pd. Subsequently, all of 
them were separately reduced at $150^{\circ} \mathrm{C}$ for 1 hour. Finally, 5 $\mathrm{mL}$ volume of PAA-AgNPs (from "ii") and $5 \mathrm{~mL}$ of PAA-Pd NPs (from "iii”) dropwise added into $10 \mathrm{~mL}$ PAH-AuNPs solution (from "I") under stirring. After the mixing, it continues further stirring for $30 \mathrm{~min}$ and the final product indicates as $\mathrm{PEC}-\mathrm{Au}_{50} / \mathrm{Ag}_{25} / \mathrm{Pd}_{25}$. For the bi-metallic $\mathrm{PEC}-\mathrm{Au}_{50} / \mathrm{Ag}_{50}$, equal volume of PAA-AgNPs and PAH-AuNPs was mixed.

Catalytic test and SERS measurement study: The catalytic performances were tested for reduction of 4-NPh by $\mathrm{NaBH}_{4}$ in the presence of multi-metallic NP-embedded PECs. $2 \mathrm{~mL}$ of $\mathrm{NaBH}_{4}(5 \mathrm{mM})$ was mixed with $0.4 \mathrm{~mL}$ of $4-\mathrm{NPh}(0.3$ $\mathrm{mM})$ in a standard quartz cell $(1 \times 1 \mathrm{~cm})$, and $0.2 \mathrm{~mL}$ of the corresponding metal NP-embedded PECs was added to the mixture. The reduction kinetics was measured by recording the UV-vis absorption spectra after constant time interval. For SERS measurements of the multi-metallic NP-embedded PECs and 4-ATP, a total of $100 \mu \mathrm{L}$ of the metallic PECs solution was dropped onto a glass substrate and dried on air. Then, $50 \mu \mathrm{L}$ of ethanol solution containing $0.25 \mathrm{mM} 4$-ATP was dropped onto the metallic PECs-coated glass substrate; the coated substrate was dried and washed. Finally, SERS spectra were recorded under a laser beam accumulation time of $5 \mathrm{sec}$ and $3 \mathrm{~mW}$ of laser power with an excitation wavelength of $632.8 \mathrm{~nm}$.

Characterization: For characterization, all samples were centrifuged and washed copiously using deinonized water to remove any unbound materials or particles. The multi-metallic NP-PECs were characterized by TEM, STEM and EDX using JEOL JEM 2100F. $\zeta$-potential measurements were carried out on a Malvern Nano-ZS zetasizer. UV-vis absorption spectra were recorded by UV-vis-NIR spectrophotometer (Shimadzu UV-3600). SERS spectra were recorded by Nanofinder 30, Tokyo Instruments with a laser excitation wavelength of $632.8 \mathrm{~nm}$ (He/Ne laser).

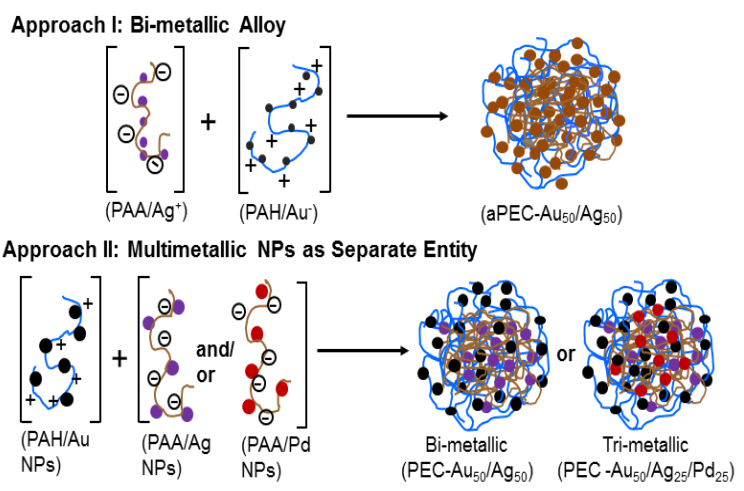

Fig. 1. Scheme for the synthesis of multi-metallic NP-embedded PECs. Subscripts indicate the mixing ratio of metal NP-loaded PEs.

\section{RESUlTS AND DISCUSSION}

Structural analysis of bimetallic alloy: Two kinds of weak PEs, PAH, $1 \mathrm{mg} / \mathrm{mL}$ (at $\mathrm{pH}=8.5$ ) and PAA, $0.40 \mathrm{mg} / \mathrm{mL}$ (at $\mathrm{pH}=4.5)$ have been used in nonstchiometric ratio $(\mathrm{PAH} / \mathrm{PAA}=1: 0.40)$ for the synthesis of PECs particle. The nonstchiometric, stable and spherical PECs particles were prepared by dropwise addition into a $10 \mathrm{~mL}$ of PAA aqueous solutions into equal volume of PAH solution under intensive stirring for $30 \mathrm{~min}$. at room temperature (Fig. 2a). Moreover, we attempted to apply PECs as a nanoreactor for the synthesis of bi-metallic alloy and multi-metallic heterostructured materials. As shown in Fig. 1, well stable, bimetallic $\mathrm{Au} / \mathrm{Ag}$ alloy-PECs were prepared by thermal reduction (Approach I). Approach II presents individually-embedded bi- and tetra-metallic PECs by mixing of PAH and PAA chains loaded monometallic NPs obtained by their post reduction mixing and stirring.

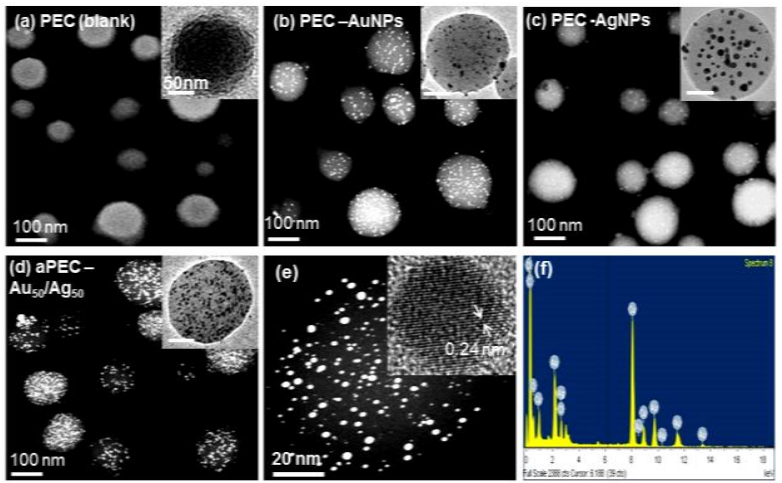

Fig. 2. STEM images of (a) PECs, (b and c) momo- and (d and e) bi-metallic alloy NPs containing aPECs-Au50/Ag50 with TEM inset images. Inset of (e) shows interplanar distance on NPs with lattice fringes. (f) EDX spectrum of (e).
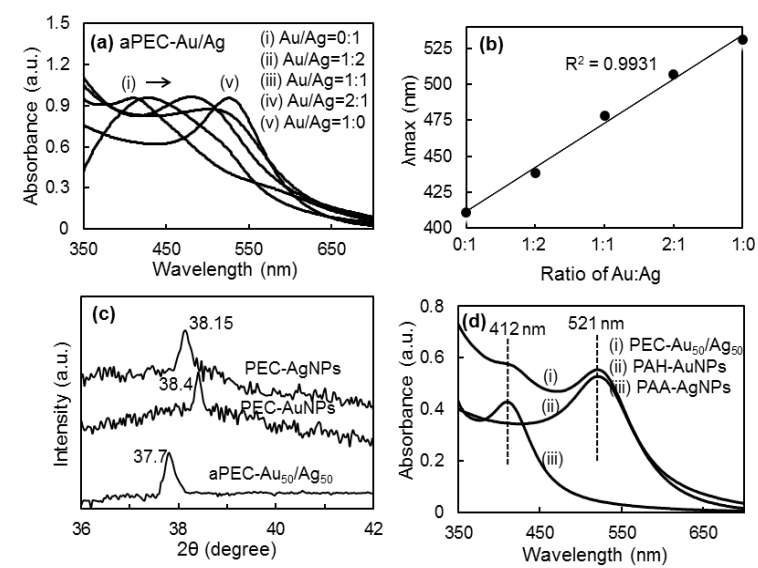

Fig. 3. (a) UV-vis absorbance spectra of aPECs-Au/Ag and (b) the correlation curve of wavelength of corresponding to the maximum absorbance for varying fractions of Au and AgNPs. (c) XRD pattern of (111) planes of $\mathrm{Ag}, \mathrm{Au}$ and $\mathrm{Au} / \mathrm{Ag}$ alloy NPs. (d) UV-vis spectra of the PAH-AuNPs, PAA-AgNPs and their post reduction mixture i.e. PEC-Au50/Ag50.

Fig. 2(a-c) exhibits STEM images of as-prepared PECs (blank), PEC-AuNPs and PEC-AgNPs respectively. Fig. 2 (d-f) displays well-distributed $\mathrm{Au} / \mathrm{Ag}$ alloy NPs with 5.31 $\pm 1.2 \mathrm{~nm}$ sizes embedded in the PECs. The EDX spectra (Fig. 2f) confirm the existence of AuNPs and AgNPs. HRTEM analysis (inset of Fig. 2e) shows clear lattice fringes of the NPs with an interplanar distance with lattice fringes $0.24 \mathrm{~nm}$ corresponding to (111) plane, which indicates that the PECs supported NPs are well crystalline [10]. To understand the dynamics of the formation of alloy NPs, UV-Vis spectra were recorded for a solution with equal concentration but varying molar ratio of both metal precursors. It is reported that in case of alloy NPs, plasmon band shifts toward another one (either blue or red) if the molar ratio of one increase by keeping constant of another precursors [10]. The mono-metallic PEC-AuNPs showed characteristic peak at $523 \mathrm{~nm}$ (Fig. 3a). Absorbance peak shows blue shift in a linear fashion from 
$509 \mathrm{~nm}$ to $431 \mathrm{~nm}$ as the ratio of Ag increases, and finally it shows a peak at $412 \mathrm{~nm}$ only for PEC-AgNPs. Plotted curve of the wavelength of those corresponding shown in Fig. $3 b$ demonstrates the linear change of UV-Vis absorbance with increasing the composition ratio of $\mathrm{Au}$ compared to $\mathrm{Ag}$ in PEC-matrixes, which ensures the formation of alloy-typed metal NPs. Furthermore XRD measurements were performed (Fig. 3c). The XRD peaks for PEC-AuNPs, PEC-AgNPs and aPEC- $\mathrm{Au}_{50} / \mathrm{Ag}_{50}$ were identified at $38.4^{\circ}, 38.15^{\circ}$ and $37.7^{\circ}$ respectively, correspond to the (111) planes of Au and Ag. The lattice constant values of Au/Ag alloy NPs showed a lower angle-side shift $\left(2 \theta=0.7^{\circ}\right.$ respect to $\mathrm{Au}$ peak $)$, revealing an expansion of the lattice after alloy formation [11].

The driving force for the formation of PECs is mainly the electrostatic interactions between oppositely charged groups. Moreover, hydrogen bonds, ion dipole forces and hydrophobic interactions play a significant role [12]. $\mathrm{PH}$-dependent ionization behavior of PE-chains [13] was considered for preparing PEC without using any sacrificial template. The $\mathrm{pH}$ of the starting aqueous solution of PAA was 4.5 , and zeta potential values were -49.83 and $-20.57 \mathrm{mV}$ for blank PAA and PAA-Ag, respectively (data not shown). Otherwise, $\mathrm{pH}$ of initial solution of $\mathrm{PAH}$ was 8.5 and calculated net charged for $\mathrm{PAH}$ was $+64.54 \mathrm{mV}$ and for PAH-Au was $+30.23 \mathrm{mV}$. Therefore, after addition of PAA-Ag (-20.57 mV) into PAH-Au(+30.23 mV), charged groups $\left(\mathrm{NH}_{3}{ }^{+}\right.$and $\left.-\mathrm{COO}^{-}\right)$of both PEs chains ionically interacted with each other and metal ions embedded spherical PEC particles were formed.
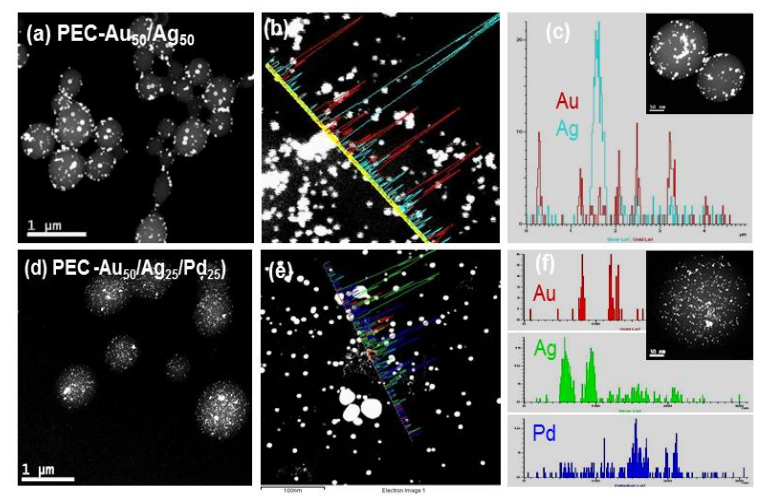

Fig. 4. STEM images for individual metal NP-embedded PECs, where (a-b) bi-, and (d-e) tri-metallic system. Corresponding line-scan EDX spectra of (c and $\mathrm{f}$ ) confirm individual metal NPs coexisting in PECs with few overlapped or phase segregated structure. Insets of (c and f) exhibit PECs used for the line-scanning.

To confirm the free active groups $\left(\mathrm{NH}_{3}{ }^{+}\right.$and $\left.-\mathrm{COO}^{-}\right)$in individual PE chains (PAH and PAA, respectively), we checked by approach II. Fig. 4 shows STEM images and line-scan EDX spectra which exhibits an existence of individual $\mathrm{Au}, \mathrm{Ag}$, and PdNPs as separate entities rather than alloy or core-shell NPs. Also in some points, overlapped/ phase segregated structure of NPs within the PECs could be found [14]. Further confirmation was carried out from UV-vis measurement (Fig. 3d(i)), where two clear peaks for $\mathrm{Au}$ and AgNPs were showen at 521 and $411 \mathrm{~nm}$, respectively after mixing equal volume of PAH-AuNPs and PAA-AgNPs. Similar individual metal peaks were observed for PEC-Au/Ag/PdNPs (data not shown).

\section{Catalytic Activities}
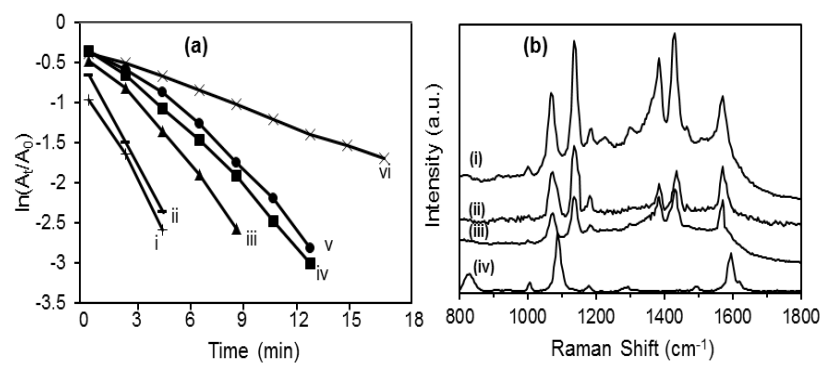

Fig.5. (a) Linear correlation of $\ln (\mathrm{At} / \mathrm{A} 0)$ versus time for the reduction of $4-\mathrm{NPh}$ to $4-\mathrm{APh}$ in the presence of ; i=tetra-metallic mixture of PEC-Au50/Ag25/Pd25; ii=aPEC-Au50/Ag50; iii=bi-metallic mixture of PEC-Au50/Ag50; iv= PEC-AuNPs; v=PEC-PdNPs; vi=PEC-AgNPs. (b) SERS spectra of 4-ATP adsorbed on the PECs embedded ; (i) tetra-metallic mixture of Au50/Ag25/Pd25; (ii) bi-metallic mixture of Au50/Ag50; (iii) aPEC-Au50/Ag50; (iv) 4-ATP as reference.

After synthesis, all materials were tested for the catalytic reduction of 4-nitrophenol (4-NPh) by $\mathrm{NaBH}_{4}$, the most intractable industrial pollutants. The reduction kinetics were monitored by UV-Vis absorption spectroscopy where the absorption of 4-NPh at $400 \mathrm{~nm}$ decreases along with a concomitant increase of the $300 \mathrm{~nm}$ peak of 4-aminophenol (4-APh) (data not shown). In pseudo-first-order kinetics a linear correlation of $\ln \left(\mathrm{A}_{\mathrm{t}} / \mathrm{A}_{0}\right)$ versus time $(\mathrm{t})$ was obtained. As shown in Table $\mathrm{I}$, the reaction proceeded very slowly by using any monometallic catalyst, whereas significantly improved by applying bimetallic alloy $\left(\mathrm{aPEC}-\mathrm{Au}_{50} / \mathrm{Ag}_{50}\right)$ and tri-metallic individual mixture of $\mathrm{PEC}-\mathrm{Au}_{50} / \mathrm{Ag}_{25} / \mathrm{Pd}_{25}$ as catalysts. This due to the modification of the electronic structure particularly related to local strain and effective atomic coordination number at the surface in multimetallic materials as well as ligand effect [15].

TABLE I: The CAlCUlated Kinetic RATE CONSTANT AND \% OF CONVERSION FOR THE CATALYTIC REDUCTION OF 4-NPH TO 4-APH USING VARIOUS COMPOSITION OF METAL ALLOY AND INDIVIDUAL MiXTURE OF

\begin{tabular}{lllllll}
\multicolumn{7}{c}{ NPS. } \\
& $\begin{array}{l}\text { PEC- } \\
\text { AuNPs }\end{array}$ & $\begin{array}{l}\text { PEC- } \\
\text { PdNPs }\end{array}$ & $\begin{array}{l}\text { PEC- } \\
\mathrm{AgNPs}\end{array}$ & $\begin{array}{l}\mathrm{aPEC} \\
\mathrm{Au}_{50} / \mathrm{Ag}_{50}\end{array}$ & $\begin{array}{l}\text { PEC- } \\
\mathrm{Au}_{50} / \mathrm{Ag}_{50}\end{array}$ & $\begin{array}{l}\mathrm{PEC}- \\
\mathrm{Au}_{50} / \mathrm{Ag}_{25} / \mathrm{Pd}_{25}\end{array}$ \\
\hline $\begin{array}{l}\text { Type of } \\
\text { catalyst }\end{array}$ & $\begin{array}{l}\text { Mono- } \\
\text { metallic }\end{array}$ & $\begin{array}{l}\text { Mono- } \\
\text { Metallic }\end{array}$ & $\begin{array}{l}\text { Mono- } \\
\text { metallic }\end{array}$ & $\begin{array}{l}\text { Bi-metallic } \\
\text { Alloy }\end{array}$ & $\begin{array}{l}\text { Bi-metallic } \\
\text { mixture }\end{array}$ & $\begin{array}{l}\text { Tri-metallic } \\
\text { mixture }\end{array}$ \\
$\begin{array}{l}\text { Rate constant } \\
\left(\mathrm{KS}^{-1}\right)\end{array}$ & $5.0 \times 10^{-3}$ & $3.94 \times 10^{-3}$ & $1.42 \times 10^{-3}$ & $7.93 \times 10^{-3}$ & $6.78 \times 10^{-3}$ & $9.83 \times 10^{-3}$ \\
$\begin{array}{l}\text { Conversion } \\
(\%)\end{array}$ & $91.3 \%$ & $94.33 \%$ & $81.6 \%$ & $94.2 \%$ & $95.4 \%$ & $96.29 \%$ \\
\hline
\end{tabular}

Finally, metal NP-embedded PECs were used for fabricating intense SERS substrates to detect different molecules. Fig. 5b displays the normal Raman spectrum of 4-ATP (line IV) and the SERS spectra of 4-ATP molecules absorbed on the surface of tri- and bi-metallic mixture and $\mathrm{Au}_{50} / \mathrm{Ag}_{50}$ alloy, (curve $\mathrm{i}$-iii). Compared with the normal Raman spectrum of 4-ATP, bands shift from $1086 \mathrm{~cm}^{-1}$ and $1594 \mathrm{~cm}^{-1}$ (line iv) to $1067 \mathrm{~cm}^{-1}$ and $1571 \mathrm{~cm}^{-1}$ (line i-iii) respectively. This indicates that thiol groups in 4-ATP directly contacts with the metal NPs modified surface by forming a strong chemical bond [16]. Two sets of bands were observed on the SERS spectra of 4-ATP on the assembling film: one set is located at $1067 \mathrm{~cm}^{-1}$, which is assigned to the 
$a_{1}$ vibration modes, and the other set is located at 1135,1383 , 1427 , and $1571 \mathrm{~cm}^{-1}$, which are assigned to the $b_{2}$ vibration modes [17]. As reported, the enhancement of $b_{2}$ modes is attributed to the chemical mechanism, most likely from the charge transfer (CT) of the adsorbate to the metal [18]. It is found that glass substrates modified by tri-metallic PEC- $\mathrm{Au}_{50} / \mathrm{Ag}_{25} / \mathrm{Pd}_{25}$ exhibits higher SERS activity. This can be explained by great number of hot spots (clearance of $\mathrm{Au} / \mathrm{Ag} / \mathrm{Pd}$ NPs aggregates), which is favorable for enhancing the SERS signals [19].

\section{CONCLUSIONS}

In summary, mono-metallic NPs and bi-metallic alloy were architecture within the PECs following a facile thermal reduction method. Also there have shown that bi-, or tri-metallic NPs coexisting as separate entities could be prepared by post reduction mixing of their constituent PE chains embedded with reduced metal NPs. The bi-metallic alloy aPEC- $\mathrm{Au}_{50} / \mathrm{Ag}_{50}$ and tri-metallic PEC- $\mathrm{Au}_{50} / \mathrm{Ag}_{25} / \mathrm{Pd}_{25}$ synergistically improved the catalytic activity compared to their monometallic $\mathrm{Au}, \mathrm{Ag}, \mathrm{PdNPs}$. In addition, promising SERS effect were observed on ATP adsorbed surface, especially tri-metallic $\mathrm{PEC}-\mathrm{Au}_{50} / \mathrm{Ag}_{25} / \mathrm{Pd}_{25}$ mixture, exhibiting higher efficiency than alloy materials. The present findings might open up a new avenue for the development of high performance multi-metallic nanostructured catalysts without using any extra sacrificial templates such as PS, MF or silica particles.

\section{ACKNOWLEDGMENT}

This work was supported by the Korea Basic Science Institute (KBSI) grant $\mathrm{T} 33740$ and a grant from the cooperative $R \& D$ program funded by the Korea Research Council for Industrial Science and Technology.

\section{REFERENCES}

[1] D. S. Wang and Y. D. Li, "Bimetallic nanocrystals: liquid-phase synthesis and catalytic applications,"Adv. Mater, vol. 23, pp.1044-1060, March 2011.

[2] L. Zhang, M. Zheng, X. Liu, and J. Sun, "Layer-by-layer assembly of salt-containing polyelectrolyte complexes for the fabrication of dewetting-induced porous coatings," Langmuir, vol. 27, pp.1346-1352, Feb. 2011

[3] W. S. Choi, H. Y. Koo, J. Y. Kim, and W. T. S. Huck, "Collective behavior of magnetic nanoparticles in polyelectrolyte brushes," $A d v$. Mate., vol. 20, pp.4504-4508, Dec. 2008.

[4] M. Dierendonck et al., "Single-step formation of degradable intracellular biomolecule microreactors," ACSNANO, vol. 5, pp. 6886-6893, Sept. 2011.

[5] T. C. Wang, M. F. Rubner, and R. E. Cohen, "Polyelectrolyte multilayer nanoreactors for preparing silver nanoparticle composites: controlling metal concentration and nanoparticle size," Langmuir, vol. 18, pp. 3370-3375, April 2002.

[6] J. Zhang et al., "Fabrication of lactobionic-loaded chitosan microcapsules as potential drug carriers targeting the liver," Acta Biomater., vol. 7, pp. 1665-1673, April 2011.

[7] U. Manna and S. Patil, "Dual drug delivery microcapsules via layer-by-layer self-assembly," Langmuir, vol. 25, pp. 10515-10522, Sept. 2009.

[8] H. Goh, H.-J. Lee, B. Nam, Y. B. Lee, and W. S. Choi., "A chemical reactor for hierarchical nanomaterials with tunable structures: a metal-triggered reaction in the confined heat chamber," Chem. Mater., vol. 23, pp. 4832-4837, Nov. 2011.

[9] D. V. Volodkin, A. I. Petrov, M. Prevot, and G. B. Sukhorukov, "Matrix polyelectrolyte microcapsules: new system for macromolecule encapsulation," Langmuir, vol. 20, pp. 3398-3406, April 2004.
[10] T. Som and B. Karmakar, "Core-shell Au-Ag nanoparticles in dielectric nanocomposites with plasmon-enhanced fluorescence: a new paradigm in antimony glasses," Nano Res, vol. 2, pp. 607-616, Aug. 2009.

[11] B. A. Kakade, T. Tamaki, H. Ohashi, and T. Yamaguchi, "Highly active bimetallic $\mathrm{PdPt}$ and $\mathrm{CoPt}$ nanocrystals for methanol electro-oxidation," J. Phys. Chem. C., vol. 116, pp. 7464-7470, April 2012.

[12] A. V. Kabanov, T. K. Bronich, V. A. Kabanov, K. Yu, and A. Eisenberg, "Soluble stoichiometric complexes from $\operatorname{poly}(N$-ethyl-4-vinylpyridinium) cations and poly(ethylene oxide)-block-polymethacrylate anions," Macromolecules, vol. 29, pp. 6797-6802, Oct. 1996.

[13] S. S. Shiratori and M. F. Rubner, "Ph-Dependent thickness behavior of sequentially adsorbed layers of weak polyelectrolytes," Macromolecules, vol. 33, pp. 4213-4219, May 2000.

[14] B. N. Wanjala et al., "Nanoscale alloying, phase-segregation, and core-shell evolution of gold-platinum nanoparticles and their electrocatalytic effect on oxygen reduction reaction," Chem. Mater., vol. 22, pp. 4282-4294, July 2010.

[15] Y. Deng et al., "Multifunctional mesoporous composite microspheres with well-designed nanostructure: a highly integrated catalyst system," J. Am. Chem. Soc., vol. 132, pp. 8466-8473, June 2010.

[16] G. Wei et al., "DNA-Network-Templated self-assembly of silver nanoparticles and their application in surface-enhanced raman scattering," J. Phys. Chem. B, vol. 109, pp. 23941-23947, Dec. 2005.

[17] J. Zheng, X. Li, R. Gu, and T. Lu, "Comparison of the surface properties of the assembled silver nanoparticle electrode and roughened silver electrode," J. Phys. Chem. B, vol. 106, pp. 1019-1023, Feb. 2002.

[18] M. Osawa, N. Matsuda, K. Yoshi, and L. Uchida, "Charge transfer resonance Raman process in surface-enhanced Raman scattering from p-aminothiophenol adsorbed on silver: Herzberg-Teller contribution," J. phys. chem., vol. 98, pp. 12702-12707, Dec. 1994.

[19] T. Wang, X. Hu, and S. Dong, "A renewable SERS substrate prepared by cyclic depositing and stripping of silver Shells on gold nanoparticle microtubes," Small, vol. 4, pp.781-786, June 2008.

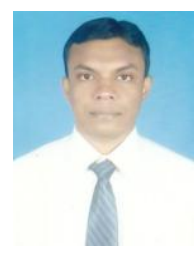

Md. Shahinul Islam received his B.Sc. in 2003 and MS.c. in 2005 in Department of Biochemistry \& Molecular Biology at Rajshahi University Bangladesh, and he got his Ph.D. in 2011 in Department of Chemistry from Chonbuk National University, Republic of Korea. He worked as a lecturer from 2005 to 2008 at Department of Arts \& Sciences for Ahsanullah University of Science \& Technology, Bangladesh. Presently, he is serving as a postdoctoral research fellow at Jeonju branch of Korea Basic Science Institute (KBSI) since September 2011. His research interest is synthesis and applications of nanostructured materials.

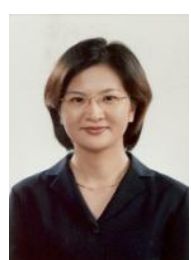

Ha-Jin Lee received her B.S. in 1995, M.S. in 1997 and Ph.D. in 2001 in Department of Chemistry at Seoul Women's University. She carried out her postdoctoral research at Institute for Molecular Science, Okazaji in Japan from 2002 to 2003 and National Institute of Standards and Technology, MD in USA from 2004 to 2005. She is now working at Jeonju branch of Korea Basic Science Institute from September of 2009 as a principal research scientist. She has published more than 60 scientific papers and her research interests mainly include development of functional nanostructures using nanocarbons and organic materials.

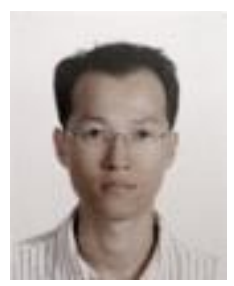

Won San Choi received his B.S. in 2000 and M.S. in 2002 in Department of Polymer Engineering at Chonnam National University, and his Ph.D. in 2006 in Department of Materials Science and Engineering from Gwangju Institute of Science and Technology (GIST). He carried out postdoctoral research at Department of Chemistry, University of Cambridge from 2006 to 2008. He worked for Korea Basic Science Institute (KBSI) from 2008 to 2011. He is a professor in the Department of Chemical and Biological Engineering at Hanbat National University since 2011. He has published more than 40 scientific papers. His research interests mainly include synthesis and application of smart and hierarchical nanostructured materials. 